\title{
Natural convection around a 3D hotstrip simulated by BEM
}

\author{
J. Ravnik \& L. Škerget \\ University of Maribor, Faculty of Mechanical Engineering, Slovenia
}

\begin{abstract}
A 3D viscous flow solver was developed using a single domain and subdomain boundary element method. The solver was accelerated using wavelet compression and the fast multipole method.

In this paper we present results of a natural convection simulation around a hotstrip. The hotstrip is located in a cavity filled with air, its height is one half of the cavities height. The flow generated by the hotstrip source is analysed in terms of flow structures and heat transfer. The position of the hotstrip is varied, its influence of the flow and heat transfer is also analysed. The problem was simulated for air $(\operatorname{Pr}=0.71)$ and for Rayleigh number values up to $R a=3.15 \cdot 10^{5}$. The results were compared with PIV measurements done by Corvaro and Paroncini.

The results show, that the hotstrip generates two large cylindrical vortical structures, one on each side as well as two small vortices on top of the hotstrip. The size of the vortices is related to the position of the hotstrip within the cavity. Heat transfer analyses show that the heat flux is largest on the sides of the hotstrip, while through the top heat losses are small.

Keywords: boundary element method, adaptive cross approximation, wavelet transform, hotstrip.
\end{abstract}

\section{Introduction}

Usage of the Boundary Element Method for simulation of fluid flow and heat transfer problems is, due to the nonlinearity and non-homogeneity of the governing equations, very challenging. Recently, we developed a 3D BEM based numerical method capable of simulation laminar viscous flow and heat transfer using a combination of single-domain BEM, subdomain BEM, wavelet transform and fast multipole method (Ravnik et al. [2-5]). The method solves the velocity vorticity 
formulation of Navier-Stokes equations and was validated using benchmark problems such as 3D channel flow, lid driven cavity flow and natural convection in a cavity.

The governing equations of the velocity vorticity formulation of Navier-Stokes equations are the kinematics equation, the vorticity transport equation and the energy transport equation. The unknown fields are the velocity field, the vorticity field and the temperature field. The boundary conditions for the velocity and temperature fields are prescribed by the user and can be of Dirichlet or Neumann type. The developed algorithm calculates boundary values of vorticity using fast multipole accelerated single domain BEM on the kinematics equation. These values serve as Dirichlet type boundary conditions for the vorticity transport equation. The transport equations are solved by sub-domain BEM. The equations are solved repeatedly in a under-relaxed nonlinear loop until convergence is achieved.

In this paper, we present results of a simulation of natural convection. Study of natural convection phenomena is important because it is used in many engineering applications, such as cooling of electronic circuits and conditioning of air. Furthermore, natural convection does not depend on any electronic or mechanical equipment and is thus very cheap. For these reasons, natural convection a subject of constant investigation by both numerical and experimental techniques (Oztop and Bilgen [6], Aydin and Yang [7]).

The source of natural convection, simulated in this paper, is a hotstrip located in a cavity filled with air. Its height is one half of the cavities height. The flow generated by the hotstrip source is analysed in terms of flow structures and heat transfer. Influence of the position of the hotstrip on flow and heat transfer is also analysed. The problem was simulated for air $(P r=0.71)$ and for Rayleigh number values up to $R a=3.15 \cdot 10^{5}$. The results were compared with PIV measurements done by Corvaro and Paroncini $[1,8]$.

\section{Problem description}

In this work we will consider air as the working fluid, for which density $\rho_{0}$, viscosity $\nu_{0}$ and diffusivity $\alpha_{0}$ will be considered constant. The Prandtl number will be set to $\operatorname{Pr}=\nu_{0} / \alpha_{0}=0.71$. We chose the characteristic length scale to be the width of the enclosure $H$. The Rayleigh $R a$ number, which is the only parameter in the non-dimensional governing equations, is defined as:

$$
R a=\operatorname{Pr} \frac{g_{0} \beta_{T} H^{3} \Delta T}{\nu_{0}^{2}},
$$

where $T$ is temperature, $\Delta T$ is the temperature difference between the hot and cold walls, $g_{0}=9.81 \mathrm{~m} / \mathrm{s}^{2}$, and $\beta_{T}$ is the thermal volume expansion coefficient. The gravity force works in the negative $z$ direction. The cavity, hotstrip and the boundary conditions are shown in Figure 1.

Corvaro and Paroncini [1] preformed a 2D PIV experiment on a hotstrip problem. The width and height of their enclosure were $H=0.05 \mathrm{~m}$. The depth of the 


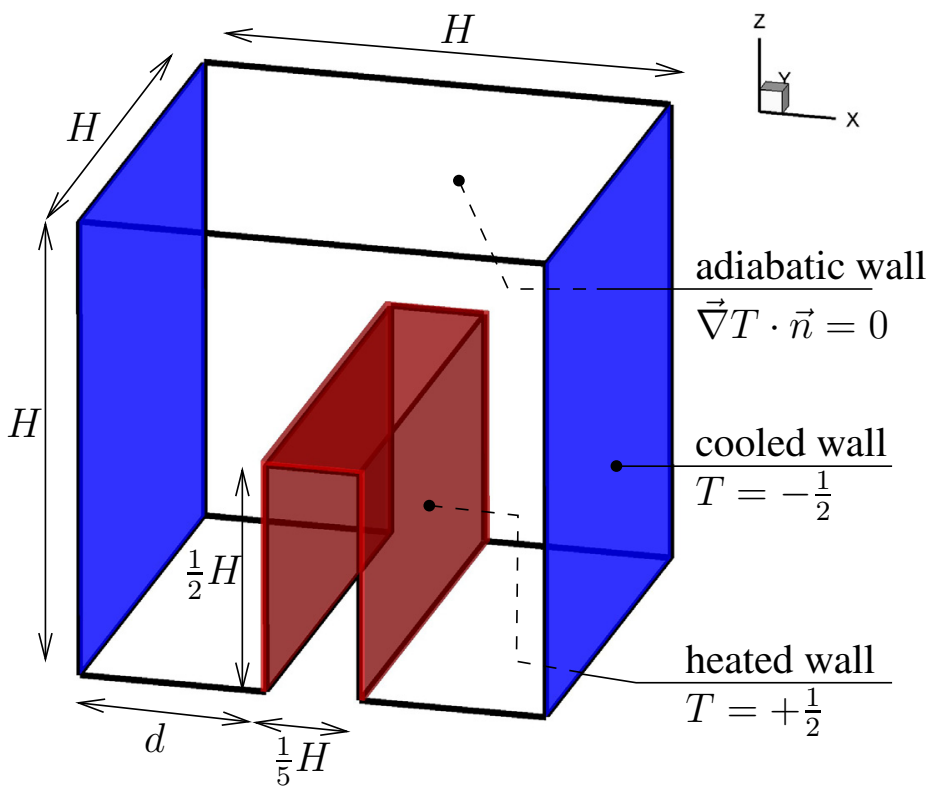

Figure 1: Geometry of the hotstrip problem and boundary conditions. Two distances of the hotstrip from the left $(x=0)$ wall were considered; $d=0.4 H$ (central position) and $d=0.5 H$ and $d=0.6 H$. The width of the hotstrip is $0.2 \mathrm{H}$ in all cases. The hotstrip is heated to $T=+0.5$, while the walls as $x=0$ and $x=H$ are cooled to $T=-0.5$. All other walls are adiabatic, i.e. there is no temperature flux through them.

enclosure was $0.42 \mathrm{~m}$, with which they achieved a $2 \mathrm{D}$ flow field. They measured the flow field in the central plane of the enclosure for Rayleigh number values between $R a=6.39 \times 10^{4}$ and $R a=3.16 \times 10^{5}$. We chose the minimum and maximum $R a$ of their experiments to compare their measurements to our simulations.

\section{Simulation results}

Since the experiment was done with a $2 \mathrm{D}$ technique measuring the flow field in the central plane of the cavity, we decided to examine the difference between the $2 \mathrm{D}$ and $3 \mathrm{D}$ cases. Thus simulations in $2 \mathrm{D}$ were preformed too. The 3D code was used to make $2 \mathrm{D}$ simulations by setting the appropriate boundary conditions and making the mesh only one element deep.

The mesh used in the 3D simulation had $20 \times 16 \times 6$ quadratic 27 -node elements with 16133 nodes, which resulted in a nonlinear system of equations with 273539 degrees of freedom. The $2 D$ mesh had $30 \times 30 \times 1$ quadratic 27 -node elements with 10173 nodes, which resulted in a nonlinear system of equations with 93732 

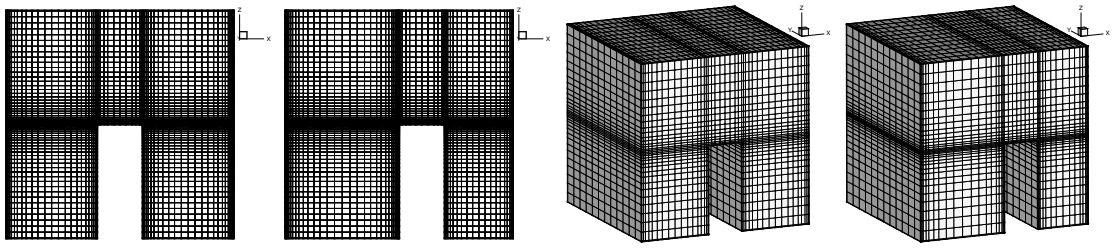

Figure 2: Presentation of the $2 \mathrm{D} 30 \times 30 \times 1$ element meshes (left) and 3D $20 \times$ $16 \times 6$ element meshes (right) for two positions of the hotstrip.
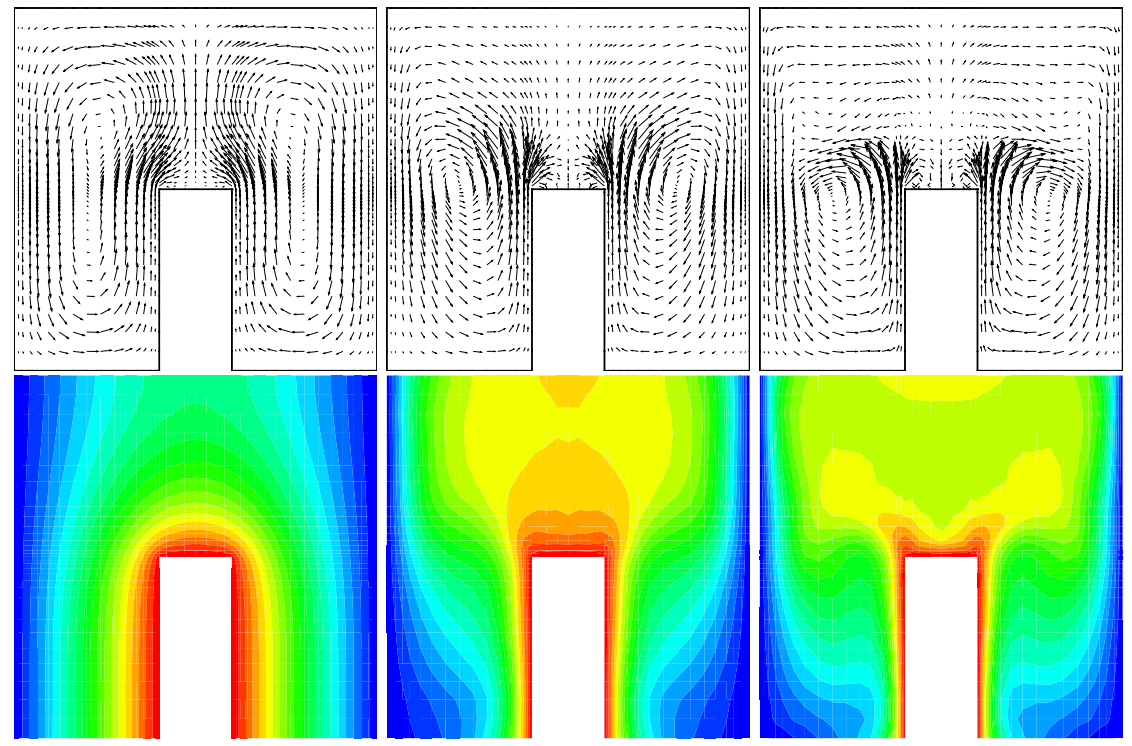

Figure 3: 3D simulation of natural convection around a hotstrip located in the central position $(d=0.4 H)$. Velocity vectors (top row) and temperature contours (bottom row) are shown on a central plane at $y=0.5 \mathrm{H}$. $R a=10^{3}$ (left), $R a=6.39 \cdot 10^{4}$ (middle), $R a=3.16 \cdot 10^{5}$ (right).

degrees of freedom. The meshes are shown in Figure 2.

\subsection{Hotstrip located in the centre of the cavity}

Figure 3 shows the velocity vectors and temperature contours of the $3 \mathrm{D}$ simulation, where the hotstrip was located in the centre of the cavity $(d=0.4 H)$, in the $y=$ $0.5 \mathrm{H}$ plane for $R a=10^{3}, R a=6.39 \cdot 10^{4}$ and $R a=3.16 \cdot 10^{5}$. The hotstrip heats the surrounding air inducing two main vortices - one on each side of the hotstrip. At the top of the hotstrip, two smaller vortices are located. They keep the hot air close to the top of the hotstrip, making heat transfer from the top of the hotstrip small compared to the heat transfer from the sides of the hotstrip. Hot air from 
Table 1: Heat transfer presented in terms of the $N u$ number value for hotstrip in the central position $d=0.4 H .2 \mathrm{D}$ and $3 \mathrm{D}$ results are presented for a range of Rayleigh number values.

\begin{tabular}{llcccc}
\hline$R a$ & $x=H$ & $x=0$ & $x=0.4 H$ & $z=0.5 H$ & $x=0.6 H$ \\
\hline \multicolumn{5}{c}{$2 \mathrm{D}$} \\
\hline $10^{3}$ & -1.8980 & -1.8980 & 1.5000 & 0.7766 & 1.5000 \\
$10^{4}$ & -2.1506 & -2.1506 & 1.8592 & 0.5665 & 1.8592 \\
$6.39 \times 10^{4}$ & -3.8125 & -3.8125 & 3.5326 & 0.5575 & 3.5326 \\
$10^{5}$ & -4.3368 & -4.3368 & 4.0399 & 0.5958 & 4.0399 \\
$3.16 \times 10^{5}$ & -5.7959 & -5.7959 & 5.4640 & 0.6675 & 5.4640 \\
\hline \multicolumn{5}{c}{$3 \mathrm{D}$} \\
\hline $10^{3}$ & -1.8958 & -1.8958 & 1.5029 & 0.7782 & 1.5029 \\
$10^{4}$ & -2.1276 & -2.1276 & 1.8377 & 0.5729 & 1.8377 \\
$6.39 \times 10^{4}$ & -3.6164 & -3.6164 & 3.3752 & 0.4846 & 3.3752 \\
$10^{5}$ & -4.1205 & -4.1205 & 3.8558 & 0.5320 & 3.8558 \\
$3.16 \times 10^{5}$ & -5.6071 & -5.6071 & 5.2333 & 0.7473 & 5.2333 \\
\hline
\end{tabular}

the sides of the hotstrip is transported upwards by convection making the thermal boundary layer thin and thus resulting in high heat transfer. At $R a=10^{3}$ the centre of the main vortices is above the hotstrip. As the Rayleigh number increases, the centre of the main vortices moves downward. At $R a=3.16 \cdot 10^{5}$ it is located by the side of the vertical hotstrip walls.

Table 1 presents Nusselt number values, as a non-dimensional measure of heat transfer, for all walls within the cavity as a function of the Rayleigh number. Heat losses through the cold walls located in planes $x=H$ and $x=0$ are represented with negative values. Heat flux emitted by the side walls of the hotstrip located in planes $x=0.4 H$ and $x=0.6 H$ is shown along with the heat flux through the top hotstrip wall located as $z=0.5 \mathrm{H}$. When the hotstrip is located in the centre of the cavity the resulting temperature and flow fields for the Rayleigh number values considered is steady and symmetrical across the $x=0.5 \mathrm{H}$ plane. Thus heat transfer through both cold walls of the cavity takes equal values, as well as heat flux coming through both side wall of the hotstrip. The appearance of the small vortices at the top of the hotstrip makes heat flux through the top of the hotstrip small. The ratio between heat flux through the side and through the top of the hotstrip increases with increasing Rayleigh number and reaches a value of approximately 10 for the highest Rayleigh number considered. 


\subsection{Hotstrip located off-centre}

Next we examine the flow field and heat transfer in the case where the hotstrip is positioned in an off-central position at $d=0.5 H$ (see Figure 1 for sketch). Offcentral position of the heat source brings non-symmetry into the problem. Velocity vectors and temperature contours for this case are shown in Figure 4. Due to the non-symmetry the main vortices now have different sizes.

The left vortex is larger, while the right vortex is squeezed in the narrow region between the hotstrip and the cold wall. In contrast to the symmetrical $d=0.4 H$ case, we observe that the centres of the main vortices are not located by the side of the hotstrip at high Rayleigh numbers, but they remain above the hotstrip. Small vortices above the top of the hotstrip may be found in this case as well. They limit the heat flux from the top of the hotstrip.

Table 2 presents heat fluxes through the cold and hot wall in the cavity. Due to non-symmetry of the problem, the heat fluxes are also non-symmetrical. Since the hotstrip is located closer to the right $(x=H)$ wall the temperature gradients are larger at this wall. Thus more heat is dissipated through the right $(x=H)$ wall than through the left $(x=0)$ wall. This fact is true for the low Rayleigh number values $R a \leq 10^{4}$. At $R a \approx \times 10^{5}$ the heat losses through both cold walls are approximately equal. The difference in heat losses through the left and right cold
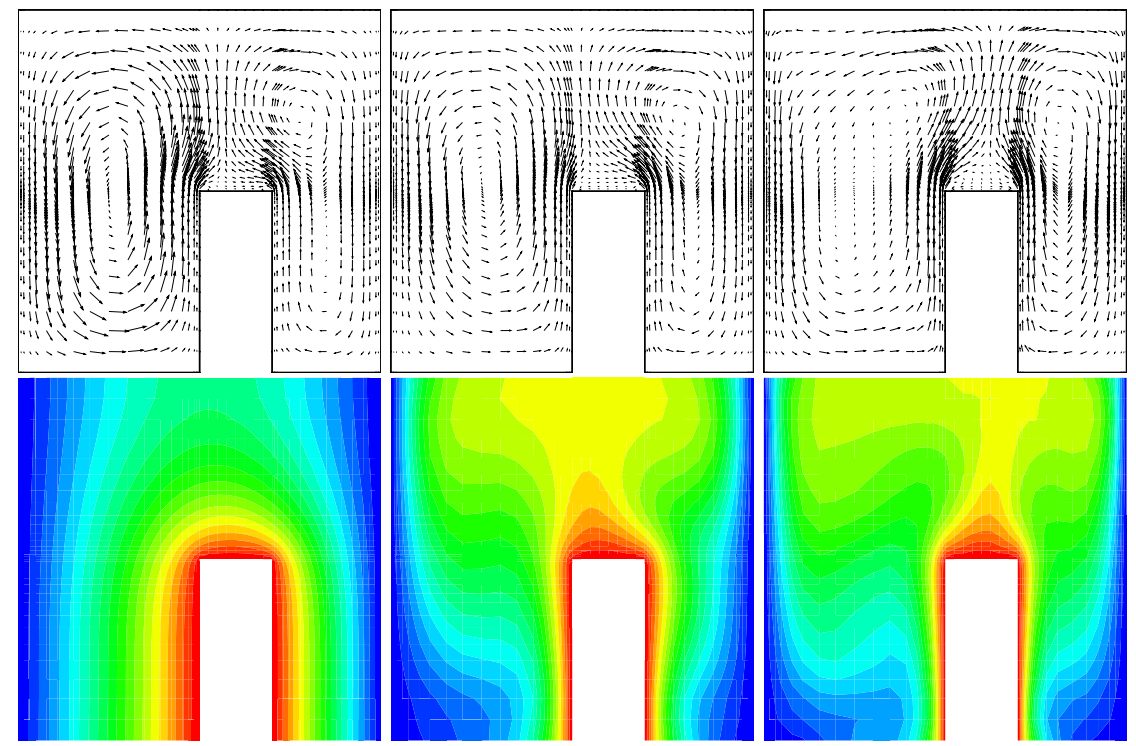

Figure 4: 3D simulation of natural convection around a hotstrip located in the offcentral position $(d=0.5 H)$. Velocity vectors (top row) and temperature contours (bottom row) are shown on a central plane at $y=0.5 \mathrm{H}$. Ra $=$ $10^{3}$ (left), $R a=6.39 \cdot 10^{4}$ (middle), $R a=3.16 \cdot 10^{5}$ (right). 
Table 2: Heat transfer presented in terms of the $N u$ number value for hotstrip in the off-central position $d=0.5 H .2 \mathrm{D}$ and $3 \mathrm{D}$ results are presented for a range of Rayleigh number values.

\begin{tabular}{llllll}
\hline$R a$ & $x=H$ & $x=0$ & $x=0.5 H$ & $z=0.5 H$ & $x=0.7 H$ \\
\hline \multicolumn{5}{c}{$2 \mathrm{D}$} \\
\hline $10^{3}$ & -2.4724 & -1.5276 & 1.2793 & 0.8053 & 1.8948 \\
$10^{4}$ & -2.6334 & -2.0065 & 1.8784 & 0.6682 & 2.0746 \\
$6.39 \times 10^{4}$ & -3.6594 & -3.8080 & 3.4966 & 0.6607 & 3.3074 \\
$10^{5}$ & -4.1692 & -4.3359 & 3.9408 & 0.6606 & 3.9069 \\
$3.16 \times 10^{5}$ & -5.6877 & -5.8030 & 5.2297 & 0.6980 & 5.5734 \\
\hline \multicolumn{5}{c}{$3 \mathrm{D}$} \\
\hline $10^{3}$ & -2.4685 & -1.5239 & 1.2840 & 0.8004 & 1.9020 \\
$10^{4}$ & -2.6214 & -1.9422 & 1.8311 & 0.6515 & 2.0757 \\
$6.39 \times 10^{4}$ & -3.6356 & -3.6338 & 3.4109 & 0.6285 & 3.2355 \\
$10^{5}$ & -4.1423 & -4.1362 & 3.8613 & 0.6176 & 3.8065 \\
$3.16 \times 10^{5}$ & -5.7560 & -5.6026 & 5.1694 & 0.7171 & 5.4808 \\
\hline
\end{tabular}

walls is more prominent at low Rayleigh number values and at high $R a$. The reason for this phenomena can be explained by looking at the flow field in Figure 4. At high $R a$ values, the right main vortex moves over the top of the hotstrip, while at low $R a$ it remains in the narrow space between the hotstrip and the wall. When the main vortex is between the side of the hotstrip and the cold wall, it results in high temperature gradients along the walls and thus causes high heat flux. This does not happen in the case of high $R a$ value.

\subsection{Small vortices on top of the hotstrip}

A unique feature of the considered problem is the existence of the small vortices on top of the hotstrip. These vortices keep the hot air above the hotstrip limiting the heat transfer. A detail of flow streamlines above the hotstrip is shown in Figure 5. Since the flow fields are steady and symmetrical when the hotstrip is located in the centre of the cavity we observe the existence of two symmetrical vortices on top of the hotstrip. The size of the vortices is very small at $R a=10^{3}$ and grows with increasing Rayleigh number. This symmetry is lost in the case, when the hotstrip is located off-centre. We observe that for lower Rayleigh values the right vortex, i.e. the vortex on the side which is closest to the cold wall, is larger than the left vortex. 


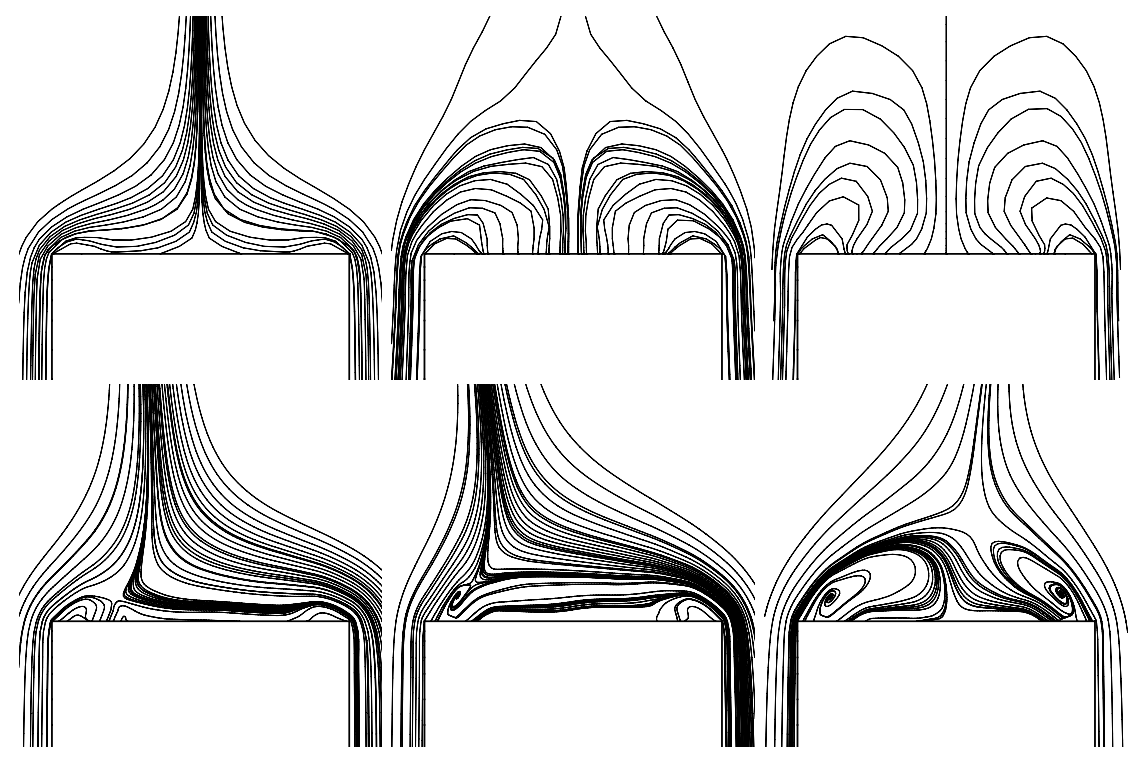

Figure 5: 3D simulation of natural convection around a hotstrip. Streamlines around the top of the hotstrip are shown for $d=0.4 H$ (top row) and $d=0.5 H$ (bottom row). $R a=10^{3}$ (left), $R a=6.39 \cdot 10^{4}$ (middle), $R a=3.16 \cdot 10^{5}$ (right).

At the highest Rayleigh number, the vortices seem approximately equally large, but from their shape, it is evident that the symmetry is lost. Due to inherent physical instability of air being heated from below, steady solution of this problem may not be sufficient. If the Rayleigh number would be higher, the problem should be simulated unsteadily. The existence of small vortices was confirmed by experiment by Corvaro and Paroncini [1].

\subsection{Comparison between 2D and 3D simulation}

Three dimensional structure of the flow field is shown in Figure 6. We present isosurfaces of the $y$ component of the velocity field. The $y$ component is directed normal to the main vortical motion, i.e. it is the direction that the $2 \mathrm{D}$ case considers to be irrelevant. We observe that the $v_{y}$ is indeed small compared to the velocities in other two directions in the low $R a$ case. But when the Rayleigh number is increased, the flow in the $y$ direction is not to be neglected. Comparing the heat transfer between the 2D and 3D cases, given in Tables 1 and 2, this conclusion is confirmed. We see, that the Nusselt number obtained with the 2D simulation are close to the 3D values for low Rayleigh number values. As the Rayleigh number increases, the difference between the 2D and 3D Nusselt number prediction becomes larger. 

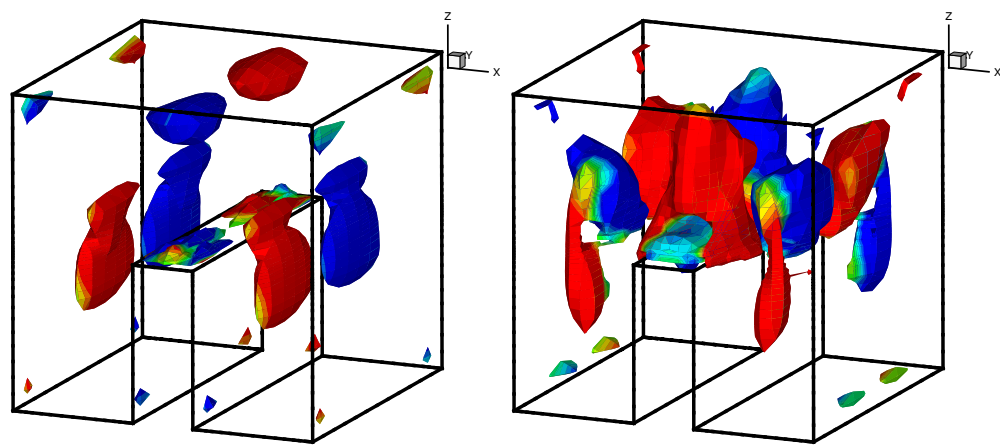

Figure 6: 3D simulation of natural convection around a hotstrip located in the central position $(d=0.4 H)$. Isosurfaces of $y$ velocity component are shown. $\left|\vec{v}_{y}\right| /|\vec{v}|=0.1, R a=10^{3}$ (left), $\left|\vec{v}_{y}\right| /|\vec{v}|=0.5, R a=3.16 \cdot 10^{5}$ (right). Color indicates $v_{y}$ direction - blue is negative, red is positive.

\section{Conclusions}

A boundary element based code was use to simulate laminar viscous fluid flow and heat transfer around a hotstrip in a cavity. The code is based on a combination of single domain BEM and sub-domain BEM and was accelerated using the fast multipole method.

Two cases were simulated. Firstly, the hotstrip was placed in a centre of a square cavity filled with air. Rayleigh number values, which were based on the temperature difference between the hotstrip and the cold walls, up to $R a=3.16 \cdot 10^{5}$ were considered. Flow in this case is symmetrical and steady. Two large vortices appear in the cavity accompanied by two small vortical structures on top of the hotstrip. By examining the difference between 2D and 3D simulation, we observed that as the Rayleigh number increases 3D effects soon become important.

In the second case, the hotstrip was located off-centre. This destroyed the symmetry of the solution. The main structures remained - the two large vortices, one on each side of the hotstrip, and the two small vortices on top of the hotstrip. But their size and position are no longer symmetrical and vary greatly with Rayleigh number.

Heat transfer was studied in terms of estimating wall heat fluxes. If the hotstrip is located symmetrically, then the heat fluxes on opposite walls are equal. Off-central position of the hotstrip causes non-symmetrical distribution of fluxes. Most of the heat flux is emitted from the sides of the hotstrip, only a small amount is emitted from the top. This is caused by the existence of small vortices on top of the hotstrip, which keep warm air in place.

The findings of this analysis are in accordance with the experimental results of Corvaro and Paroncini [1].The BEM based flow solved thus proved capable of simulating the flow and predicting the heat transfer. 


\section{References}

[1] Corvaro, F. \& Paroncini, M., An experimental study of natural convection in a differentially heated cavity through a 2D-PIV system. International Journal of Heat and Mass Transfer, 52, pp. 355-365, 2009.

[2] Ravnik, J., Škerget, L. \& Žunič, Z., Combined single domain and subdomain BEM for 3D laminar viscous flow. Eng Anal Bound Elem, 33, pp. 420-424, 2009.

[3] Ravnik, J., Škerget, L. \& Žunič, Z., Fast single domain-subdomain BEM algorithm for 3D incompressible fluid flow and heat transfer. Int J Numer Meth Engng, 77, pp. 1627-1645, 2009.

[4] Ravnik, J., Škerget, L. \& Žunič, Z., Comparison between wavelet and fast multipole data sparse approximations for Poisson and kinematics boundary domain integral equations. Comput Meth Appl Mech Engrg, 198, pp. 14731485, 2009.

[5] Ravnik, J., Škerget, L. \& Žunič, Z., Velocity-vorticity formulation for 3D natural convection in an inclined enclosure by BEM. Int J Heat Mass Transfer, 51, pp. 4517-4527, 2008.

[6] Oztop, H. \& Bilgen, E., Natural convection in differentially heated and partially divided square cavity with internal heat generation. Int J Heat Fluid Flow, 48, pp. 1470-1479, 2005.

[7] Aydin, O. \& Yang, W., Natural convection in enclosures with localized heating from below and symmetrical cooling from sides. Int J Num Meth Heat Fluid Flow, 10, pp. 519-529, 2000.

[8] Corvaro, F. \& Paroncini, M., A numerical and experimental analysis on the natural convective heat transfer of a small heating strip located on the floor of a square cavity. Applied Thermal Engineering, 28, pp. 25-35, 2008. 